Article

\title{
Novel Genetic Markers for Early Detection of Elevated Breast Cancer Risk in Women
}

\author{
Bohua $\mathrm{Wu}^{1}$, Yunhui Peng ${ }^{2}$, Julia Eggert ${ }^{1}$ and Emil Alexov ${ }^{1,2, * \mathbb{D}}$ \\ 1 Healthcare Genetics, School of Nursing, Clemson University, Clemson, SC 29634, USA; \\ bohua@clemson.edu (B.W.); jaegger@clemson.edu (J.E) \\ 2 Computational Biophysics and Bioinformatics, Department of Physics, Clemson University, Clemson, \\ SC 29634, USA; yunhuip@clemson.edu \\ * Correspondence: ealexov@clemson.edu; Tel.: +1-864-908-4796
}

Received: 2 September 2019; Accepted: 25 September 2019; Published: 28 September 2019

check for updates

\begin{abstract}
This study suggests that two newly discovered variants in the MSH2 gene, which codes for a DNA mismatch repair (MMR) protein, can be associated with a high risk of breast cancer. While variants in the MSH2 gene are known to be linked with an elevated cancer risk, the MSH2 gene is not a part of the standard kit for testing patients for elevated breast cancer risk. Here we used the results of genetic testing of women diagnosed with breast cancer, but who did not have variants in BRCA1 and BRCA2 genes. Instead, the test identified four variants with unknown significance (VUS) in the MSH2 gene. Here, we carried in silico analysis to develop a classifier that can distinguish pathogenic from benign mutations in $\mathrm{MSH} 2$ genes taken from ClinVar. The classifier was then used to classify VUS in MSH2 genes, and two of them, p.Ala272Val and p.Met592Val, were predicted to be pathogenic mutations. These two mutations were found in women with breast cancer who did not have mutations in BRCA1 and BRCA2 genes, and thus they are suggested to be considered as new bio-markers for the early detection of elevated breast cancer risk. However, before this is done, an in vitro validation of mutation pathogenicity is needed and, moreover, the presence of these mutations should be demonstrated in a higher number of patients or in families with breast cancer history.
\end{abstract}

Keywords: MSH2; breast cancer; personalized medicine; early diagnostics; computational modeling; disease-causing mutations; variants

\section{Introduction}

The MSH2 gene is a DNA mismatch repair (MMR) gene and its protein product has a crucial function for detecting and repairing DNA errors [NM_000251.1]. The MSH2 protein [NP_000242.1] binds two other proteins, MSH6 or MSH3, to form a protein complex. The complex's function is to identify the errors in DNA during DNA replication and recombination [1]. The MSH2/MSH6 complex recognizes base to base mismatch and small insertion/deletion errors, while the MSH2/MSH3 complex initiates repair of longer insertions and deletions [1,2]. Both complexes recruit downstream MMR proteins like MLH1/PSM2, or the MLH1/MLH2 complex to complete the repair process [1,3].

Mutations in the MSH2 gene were reported to be associated with the Lynch syndrome (LS), also known as hereditary nonpolyposis colorectal cancer (HNPPC) [4]. Mutations in the MSH2 gene were also observed in patients affected with adenocarcinoma of the colon and endometrial carcinoma [5-7]. Other researchers reported that LS is different from common colon cancer (with sporadic mutations) that has an earlier onset age [8]. Moreover, $\mathrm{MSH} 2$ mutations carriers were found to have a cumulative risk of colorectal cancer, independent of gender, based on research of 121 families with LS [9]. More than half of the LS cases were attributed to MSH2 mutations [10,11]. 
In addition to its association with colorectal cancer, $\mathrm{MSH} 2$ mutations are linked with hematological malignancy, gastrointestinal, urinary tract, ovary and other types of cancers as well [12-16]. Even more, some researchers demonstrated that $\mathrm{MSH} 2$ mutation carriers have an increased risk of breast cancer (BC) with or without a LS family history [15,17-19]. A novel MSH2 mutation, NM_000251.2:n.2781G>T, was identified in a breast cancer patient with a family history of $\mathrm{BC}$, ovarian cancer and some other Lynch syndrome (LS)-associated cancers [20]. The patient did not have mutations in commonly tested breast cancer genes, BRCA1, BRCA2, CHEK1 or RAD51C, but the same mutation in the MSH2 gene was also identified in other family members [20]. This finding prompted the need to complete genetic testing of the MSH2 gene, when $B R C A 1$ and $B R C A 2$ are without mutations, but multiple family members have BC history [20].

The MSH2 gene is located on chromosome 2p21-p16.3 with 16 exons [NM_000251.1]. The MSH2 protein contains 934 amino acids and can be grouped into five domains (Figure 1): Mismatch binding, connector, lever, clamp, and ATPase domains. Domain 1 is the mismatch binding domain, containing amino acids 1 to 124; domain 2 is the connector, comprised of amino acids from 125 to 297; domain 3 is the lever, consisting of two segments, which are the residues from 300 to 456 and residues 554 to 619; domain 4 is the clamp domain, containing the residues from 457 to 553 , which are located between the two segments of the lever domain; and domain 5 is the ATPase domain, consisting of the residues from 620 to 934 [21,22]. The MSH2 protein is expressed in the nucleus, nucleoplasm, and membrane, and is widespread within lymph nodes, testis, colon and 24 other tissues.

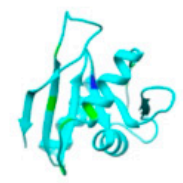

Domain 1

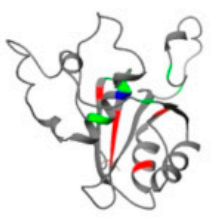

Domain 2

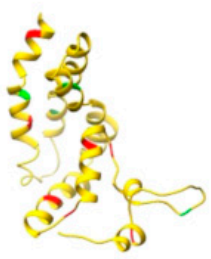

Domain 3.1

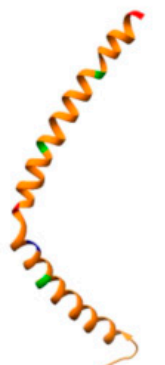

Domain 3.2

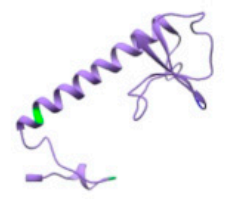

Domain 4

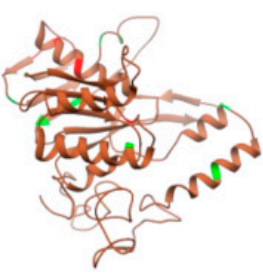

Domain 5

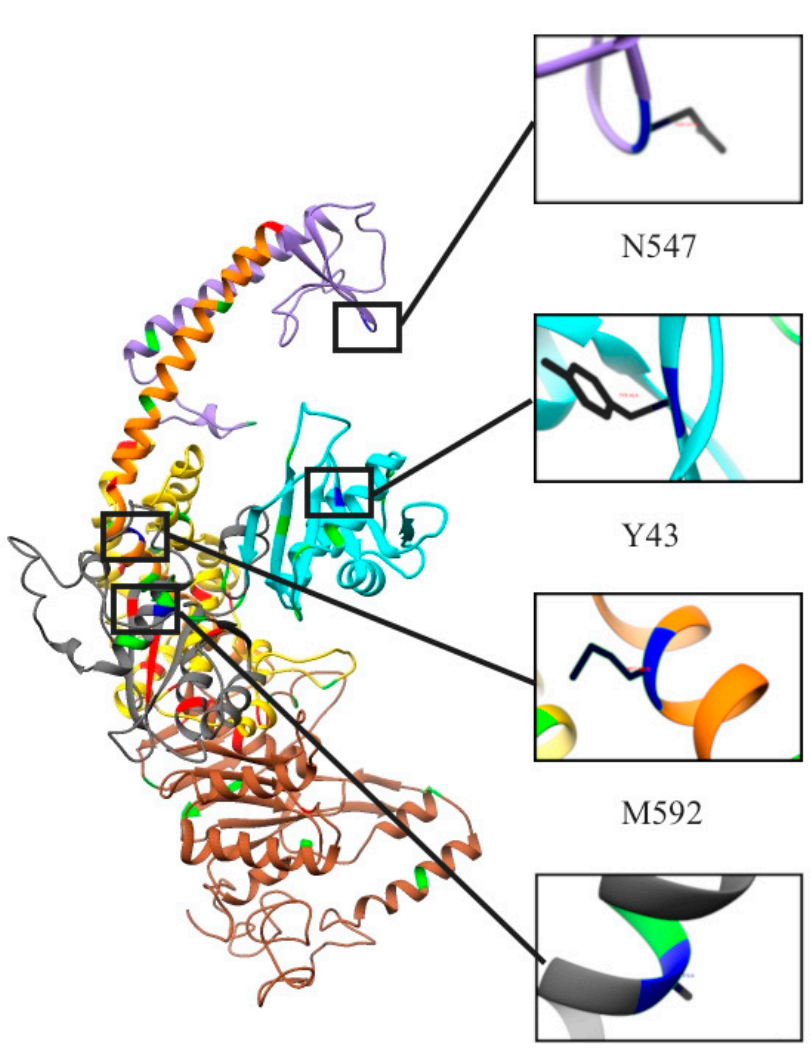

MSH2
A 272

Figure 1. The 3D Structure of the MSH2 Protein. Each domain is depicted in a different color: Domain 1(cyan) mismatch binding domain, domain 2 (dark gray) connector, domain 3.1 (yellow) levers, domain 3.1 (orange) levers, domain 4 (purple) clamps, and domain 5 (sienna) ATPase domain. Differentiation among the mutations is depicted with pathogenic in red, non-pathogenic in green and unknown significance (VUS) in blue. 
The majority of pathogenic mutations in the $\mathrm{MSH} 2$ gene has been shown to alter DNA-protein interactions, protein-protein interaction, ATPase activity, allosteric signaling between DNA and ATP binding locations, and protein stability [21,22]. Some mutations, for example: p.Val161Asp, p.Cys333Tyr, p.His639Tyr and p.Cys697Phe, were found to be deleterious, affecting allosteric signaling, and the deficiency was confirmed in vitro [21,23]. p.Ala636Pro was predicted to be pathogenic and to affect the stability of the protein structure. This result was consistent with the in vitro experiment indicating that the structure of $\mathrm{MSH} 2$ is damaged [21,23]. Mutations in the $\mathrm{MSH} 2$ gene may cause structural defects of the MSH2 protein, which typically alter the protein structure or changes binding and interactions [21]. This may explain the prevalence of the low expression level of the MSH2 protein in tumor tissues.

There are 40 pathogenic missense mutations in $\mathrm{MSH} 2$ gene reported in ClinVar, a public archive of human gene variants associated with diseases [24,25]. However, numerous variants in MSH2 are still listed in the unknown significance category. More specifically, 1238 missense mutations in the $\mathrm{MSH} 2$ are not classified. Furthermore, understanding the pathogenicity effect of $\mathrm{MSH} 2$ variants would enhance the knowledge of drug resistance and sensitivity of tumor therapy [26].

For the purpose of our investigation, variants in the $\mathrm{MSH} 2$ gene were retrieved from ClinVar [24], Exome Aggregation Consortium (EXAC), the 1000 Genomes Browser [27] and NHLBI-GO Exome Sequencing Project online databases [28]. They were grouped into "pathogenic and benign", and their effects of various MSH2 protein characteristics (as structural stability, evolutionary conservation score, etc.) were modeled. The results were used to generate a classifier that discriminates pathogenic from benign mutations. The classifier was then used to classify four mutations identified in patients with $\mathrm{BC}$ and two of them, p.Ala272Val and p.Met592Val, are predicted to be pathogenic mutations and associated with elevated $\mathrm{BC}$ risk.

\section{Results and Discussion}

To identify what physicochemical characteristics and thermodynamics parameters are essential to discriminate pathogenic versus benign mutations, we systematically examined 22 features (Table 1 ). Those 22 physicochemical characteristics were investigated for their plausible linkage with disease. Below, we outline the outcome for each of these characteristics and parameters.

Table 1. Area under the curve (AUC) value of receiver operating characteristics (ROC). Twenty-two physicochemical characteristics and thermodynamics parameters being investigated. Protein structures being analyzed: Wild-type 1; non-pathogenic mutations 41; pathogenic mutations 34 .

\begin{tabular}{cccc}
\hline Classifiers & AUC Value & Classifiers & AUC Value \\
\hline Folding free energy change & 0.77 & H-bond numbers of mutant residues & 0.54 \\
\hline Binding free energy change & 0.53 & H-bond numbers of wild-type residues & 0.52 \\
\hline Evolutionary conservation score (ECS) & 0.81 & H-bond number change upon mutations & 0.52 \\
\hline $\begin{array}{c}\text { Average root mean square deviations } \\
\text { (RMSD) of all protein structures }\end{array}$ & 0.80 & B-factors of wild-type residues & 0.69 \\
\hline $\begin{array}{c}\text { RMSD change upon mutations } \\
\text { Root mean square fluctuations (RMSF) } \\
\text { of mutant residue }\end{array}$ & 0.65 & $\begin{array}{c}\text { Relative solvent accessible surface area } \\
\text { (rSASA) of mutation residues }\end{array}$ & 0.69 \\
\hline RMSF of wild-type residue & 0.69 & rSASA of wild-type residues & 0.72 \\
\hline RMSF change upon mutations & 0.73 & rSASA change upon mutations & 0.50 \\
\hline Cumulative RMSF of mutant & 0.57 & Residue size change upon mutations & 0.70 \\
\hline $\begin{array}{c}\text { Cumulative RMSF of wild-type } \\
\text { Cumulative RMSF change upon } \\
\text { mutation }\end{array}$ & 0.61 & Residue charge change upon mutations & 0.70 \\
\hline
\end{tabular}




\subsection{Mapping All Missense Mutations onto the 3D Structure of MSH2 Protein}

The dataset contained 41 non-pathogenic mutations, 34 pathogenic mutations, and 4 VUS. Mapped onto the 3D structure of MSH2 protein, one can see that most of the non-pathogenic mutations were located on domain 1 (mismatch binding domain), domain 2 (connector domain 3.1 lever) and domain 5 (ATPase binding domain) (Figure 1). In contrast, no pathogenic mutations were located on domain 1. The largest number of mutations, both pathogenic and non-pathogenic mutations, were found on domain 5. The VUS were located in domains 1, 2, 3.2 and 4 (Figure 1). Thus, with the exception of the observation that there were no pathogenic mutations in domain 1, the rest of the MSH2 protein structure harbored mutations of all types.

\subsection{Evolutionary Conservation Score (ECS)}

The evolutionary conservation score (ECS) represents the conservation of a given residue type at the same location of the same protein in various species. A higher ECS indicates that the residue type was conserved and any mutation was expected to be unwanted. The results indicate that 156 residues have an ECS over 0.9, including 13 pathogenic mutations and four non-pathogenic mutations (Figure 2). Most of the highly conserved residues were located on domains 2, 3.1 and 5. A total of 12 out of 34 pathogenic mutations were located on domain 3.1. Fifteen locations with pathogenetic mutations $(44 \%)$ had an ECS over 0.9 and only five locations with pathogenic mutations (15\%) had an ECS lower than 0.8 (Figure 3A). In contrast, only four locations with non-pathogenic mutations $(10 \%)$ had an ECS higher than 0.9 (Figure 3A). The results showed that most of the locations with pathogenic mutations were located at highly conserved sites, while the locations with non-pathogenic mutations were not.

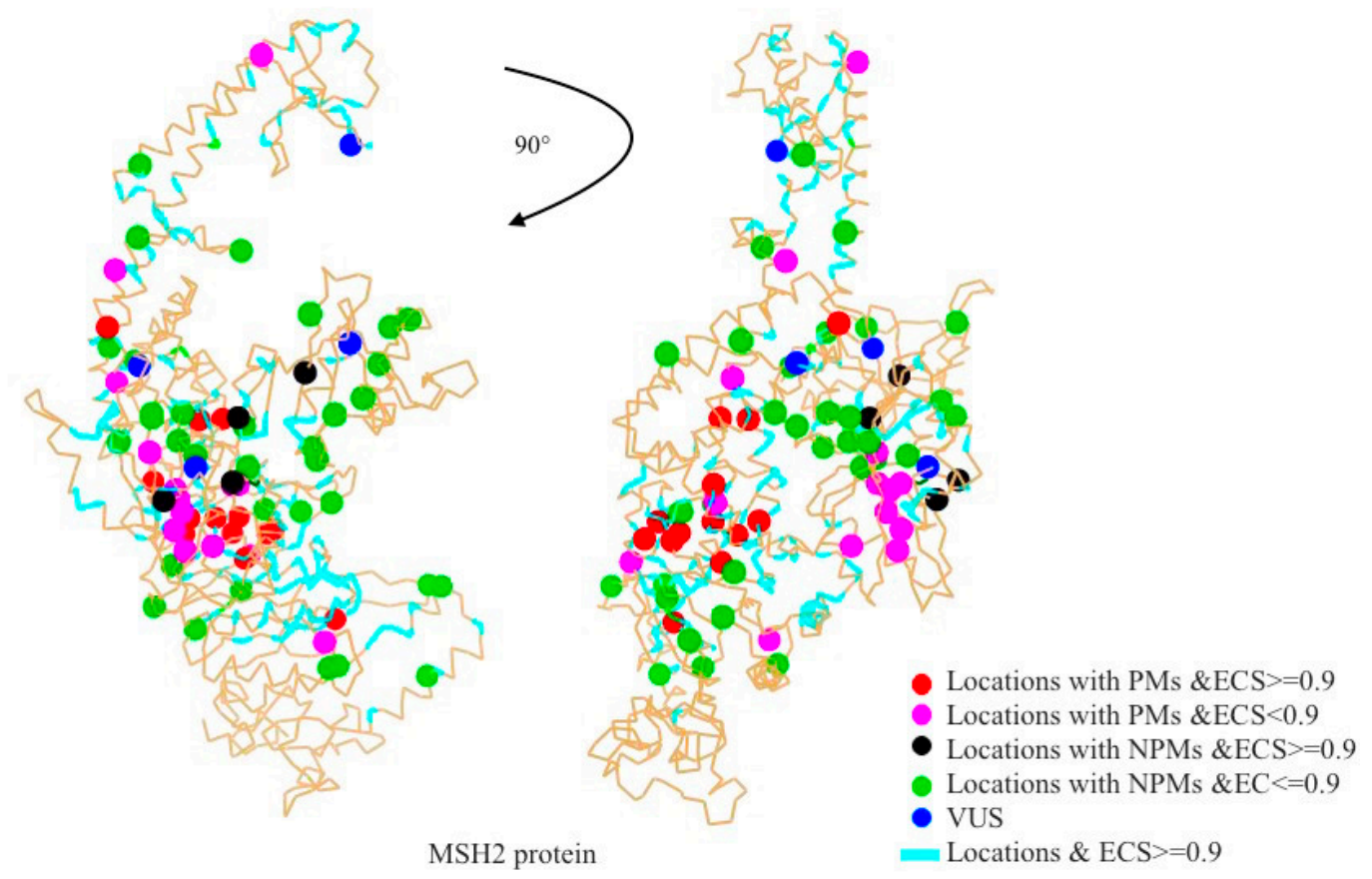

Figure 2. Visualization of highly conserved residues and mutations mapping onto the MSH2 protein structure. PMs: Pathogenic mutations, NPMs: Non-pathogenic mutations, VUS: Variants of unknown significance, and ECS: Evolutionary conservation score. 


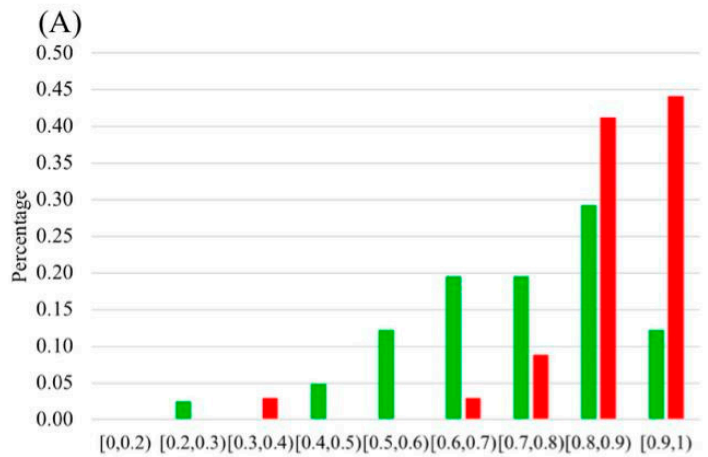

Evolutionary Conservation Score

(C)

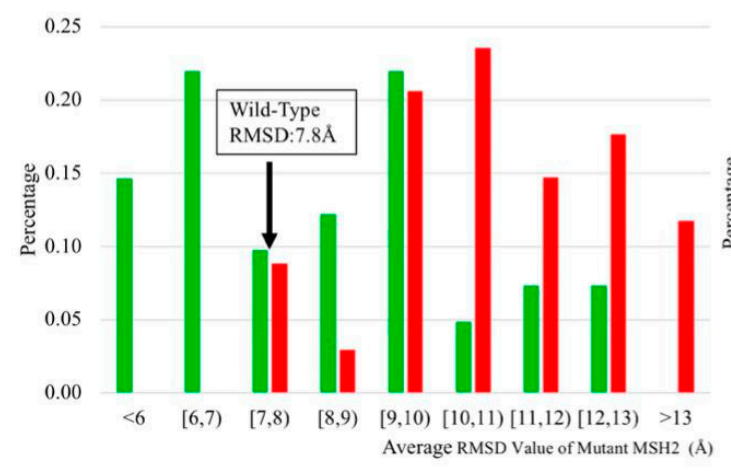

(B)
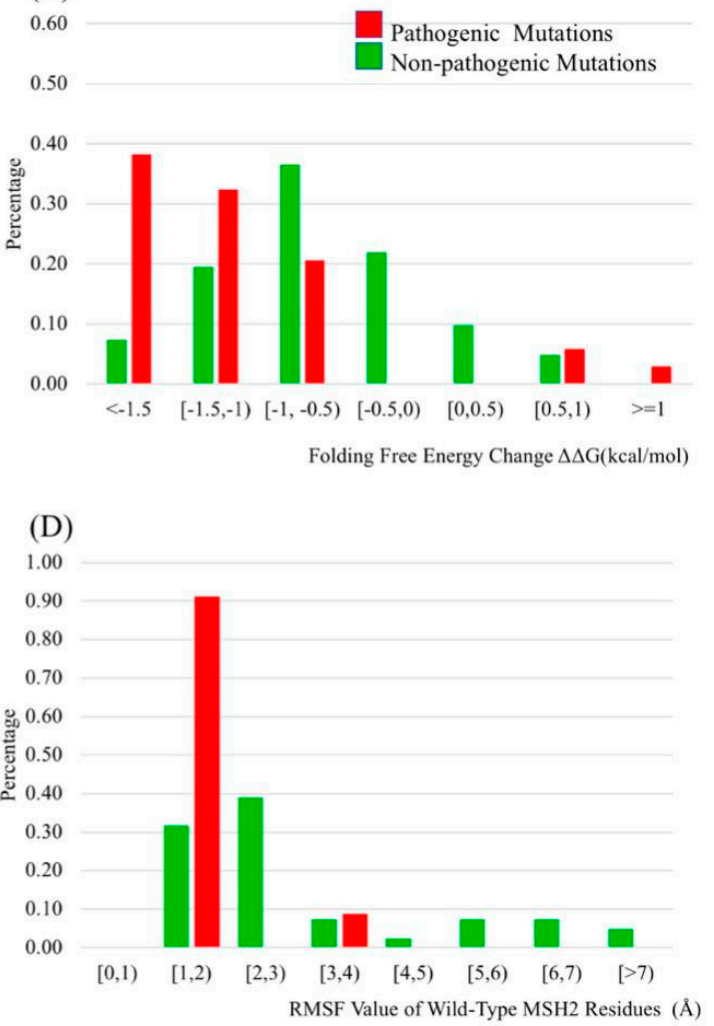

Figure 3. The distribution of evolutionary conservation score, folding free energy change upon the mutations, the average RMSD value of the MSH2 $\mathrm{C} \alpha$, and the RMSF value of the MSH2 wild-type residues. (A) There were around 2/3 of the non-pathogenic mutations with an EC score below $80 \%$; in contrast, more than $80 \%$ of pathogenic mutations have an evolutionary conservation score (ECS) higher than $80 \%$. (B) This figure demonstrates that around $30 \%$ of non-pathogenic mutations had a $\Delta \Delta \mathrm{G}$ between $-0.5 \mathrm{kcal} / \mathrm{mol}$ and $0.5 \mathrm{kcal} / \mathrm{mol}$. However, most of the pathogenic mutations had a larger $\Delta \Delta \mathrm{G}$ comparing to non-pathogenic mutations. (C) The average RMSD value of wild-type MSH2 protein was 7.8 A. Some non-pathogenic mutants had a RMSD value lower than the wild-type, but pathogenic MSH2 mutants usually have a much higher value than the wild-type. (D) The RMSF value of wild-type residues were calculated. Residues with a corresponding pathogenic mutation were labeled as red, and residues with a corresponding non-pathogenic mutation were labeled as green. More than $90 \%$ of wild-type residues on pathogenic positions had a RMSF between 1 to $2 \AA$, but most of wild-type residues on non-pathogenic positions have a larger RMSF value.

\subsection{Folding and Binding Free Energy Changes}

Folding free energy change presents the change of protein stability due to mutation(s), and the binding free energy change presents the impact of mutation(s) on protein-protein interactions. To further assess the differences between pathogenic mutations and non-pathogenic mutations, the changes of folding and binding free energy were calculated with various approaches: DUET [29], I-mutant 3.0 [30], mCSM [31], SAAFEC [32], SDM [33], PoPMuSiC [34] BeAtMuSiC [35], MutaBind [36], and SAAMBE [37].

The prediction results indicate that most non-pathogenic mutations had no effect on protein stability, but pathogenic mutations destabilized the protein structure (Figure 3B). There were 14 non-pathogenic mutations resulting in a folding energy change within $0.5 \mathrm{kcal} / \mathrm{mol}(0.5 \geq \Delta \Delta G \geq-0.5 \mathrm{kcal} / \mathrm{mol})$. Such small changes (within $0.5 \mathrm{kcal} / \mathrm{mol}$ ) were usually considered as non-significant. Comparing changes of $\Delta \Delta \mathrm{G}$ due to pathogenic and non-pathogenic mutations, the $\Delta \Delta \mathrm{G}$ changes due to pathogenic mutations were larger. None of the pathogenic mutations resulted in $\Delta \Delta \mathrm{G}$ between $-0.5 \mathrm{kcal} / \mathrm{mol}$ and $0.5 \mathrm{kcal} / \mathrm{mol}$, 
and around $80 \%$ of the $\Delta \Delta \mathrm{G}$ of pathogenic mutations were greater than $1 \mathrm{kcal} / \mathrm{mol}$ or below $-1 \mathrm{kcal} / \mathrm{mol}$ (Figure 3B). Any large change of the folding energy, making the protein less or more stable than the wild-type, was considered to be greatly affecting protein function [38,39].

The binding energy change $(\Delta \Delta \Delta \mathrm{G})$ results did not show obvious differences between pathogenic and non-pathogenic mutations (Figure S1). The prediction for non-pathogenic mutations showed most of the binding free energy changes $(\Delta \Delta \Delta \mathrm{G})$ upon non-pathogenic mutations were between $-0.5 \mathrm{kcal} / \mathrm{mol}$ and $0.5 \mathrm{kcal} / \mathrm{mol}$ with a small standard deviation. However, the prediction results made from different webservers frequently showed controversial results. All pathogenic mutations were not located on the protein-protein interface, which may explain why the binding free energy changes failed to discriminate pathogenic from non-pathogenic mutations.

\subsection{Molecular Dynamics (MD) Simulation}

All of the mutants described above, and the wild-type protein were subjected to extensive MD simulations. The simulation results showed that pathogenic and non-pathogenic mutations had different protein conformation dynamics. The root mean square deviations (RMSDs) for the C $\alpha$ of each mutant and wild-type protein were calculated (Figure 3C). Most RMSD values for non-pathogenic mutation protein were close to the value of wild-type proteins (Figure 3C). In contrast, the majority of pathogenic type proteins had an extremely large average RMSD. The larger RMSD indicated lower stability of the corresponding protein.

The root mean square fluctuations (RMSF) values of $\mathrm{C} \alpha$ were computed to determine if the wild-type or the mutated residue affected the dynamic conformation of the corresponding location of the protein. The RMSF value of each residue for wild-type and mutant type proteins were calculated in order to examine the flexibility of the protein structure at that position in the wild-type and mutant proteins. We observed that RSMF values of positions of mutations calculated on the corresponding mutant protein did not discriminate between pathogenic and non-pathogenic mutations. However, if the RMSFs were calculated with the wild-type protein, we found that $90 \%$ of pathogenic mutation locations had a RMSF value between 1-2 $\AA$, while non-pathogenic mutation locations have the opposite trend (Figure 3D). The low RMSF value implicated that the pathogenic mutation was located in a more rigid region where the residue was less flexible and had limited movement during the simulation than the non-pathogenic mutations.

A mutation may alter the flexibility and induce conformational change to nearby residues. In order to examine the regional RMSF change, the cumulative RMSF of 11 neighborhood residues were calculated by summing up the RMSF value of the position of the variant (using either wild-type or the corresponding mutant structure), and five neighborhood residues before and after the variant (with respect to the amino acid sequence). However, we did not observe any trend indicating that this quantity could discriminate between pathogenic and non-pathogenic mutations (Figure S2A,B).

\subsection{B-Factors}

B-factors provided in the corresponding Protein Data Bank (PDB) file are indicators of conformational flexibility of individual atoms. Thus, we attempted to see if B-factors at the corresponding location of the wild-type protein could potentially discriminate the pathogenic and non-pathogenic mutations. The results were not inclusive. Indeed, Figure S3 shows the plot of RMSFs versus B-factors and while one appreciates that non-pathogenic mutations were much more scattered compared with pathogenic, the signal was not strong. However, one observed that the B-factor of most pathogenic mutation positions fell between the interval of $80-85$ and data points were clustered. Thus, the pathogenic mutation locations tended to have a smaller RMSF, and B-factor values compared with non-pathogenic sites.

\subsection{Relative Solvent Accessible Surface Area (rSASA) and B-Factors}

The relative solvent accessible surface area (rSASA) for both wild-type and mutant type residue were calculated with Equation (3). The results show that 20 of 34 wild-type residues in the pathogenic 
mutation group had a rSASA under 0.1. This indicates that most of the pathogenic mutations were in buried positions in the protein structure.

The distribution of B-factors and rSASA values were examined for wild-type residues (Figure S4). Pathogenic mutations had a smaller B-factor and rSASA than non-pathogenic mutations. This finding infers that the pathogenic mutations in MSH2 involved residues that were more rigid and buried than those of non-pathogenic mutations.

\subsection{Protein Distance (PD)}

The PD (Equation (1)) was tested as a discriminator between pathogenic and non-pathogenic mutations. The results are shown in Table S1. The PD was small for most mutations in the non-pathogenic group, while PD was large for the pathogenic group (Figure S5).

\subsection{Hydrogen Bonds}

The H-bonds were calculated with Equation (4) for both mutant and the wild-type residues, and the changes of the total $\mathrm{H}$-bonds were counted. The results did not show significant differences between pathogenic and non-pathogenic groups (Figure S6A-C).

\subsection{Receiver Operating Characteristics (ROC)}

The main goal of this investigation was to identify physicochemical characteristics and thermodynamics parameters, which can discriminate between pathogenic or non-pathogenic mutations and be used as an input for a classifier (Table 1). To optimize the performance of the prediction model, these 22 physicochemical characteristics and thermodynamics parameters were analyzed with ROC (Table 1). The area under the curve (AUC) of ROC indicates whether a predictor is better than random. In developing a classifier, one wants to optimize the performance while reducing the number of input parameters (to avoid over-fitting). Thus, we selected a particular threshold for AUC to reduce the number of input parameters. The AUC results for folding free energy change, evolutionary conservation score, the average RMSD of the protein, and the RMSF of the wild-type residue position were higher than 0.75 , which made us select them as good classifiers.

\subsection{Selecting the Best Predicting Protocol}

Folding free energy change, evolutionary conservation score, the average RMSD of the protein and the RMSF of the wild-type residue were selected as classifier in K-nearest neighbors (KNN) and support vector machine (SVM) methods (see method section). The dataset, including 34 pathogenic missense mutations and 41 non-pathogenic missense mutations, was randomly allocated into a training dataset (52 mutations) and a testing dataset (23 mutations), and then subjected to KNN and SVM classification. KNN analysis was performed with or without RMSD and RMSF, and we tested different $\mathrm{K}$ values to find the optimal value of K. SVM analysis was also performed with or without RMSD/RMSF. Linear, polynomial, radial and sigmoid kernel were tested in SVM with the optimal cost and gamma value as well. The classification shows better performance without RMSD and the accuracy is $100 \%$ when the $\mathrm{K}$ value is six or eight in the KNN method. The prediction accuracy was lower in the SVM method. Finally, the KNN classification method using folding free energy change, evolutionary conservation score and the RMSF of wild-type residues was selected as the best predictor.

\subsection{Classification of VUS Using KNN Method}

The KNN method was utilized to classify the four VUS from clinical data (Table 2). It predicted that VUS p.Ala272Val and p.Met592Val were pathogenic, while p.Tyr43Cys and p.Asn547Ser were non-pathogenic. Furthermore, the classification was assessed via the RMSF, B-factors and rSASA of wild-type residues positions (Figure S4). The results showed that the corresponding quantities 
at positions 272 and 592 were into the area that most pathogenic mutations were located, while the quantities associated with positions 43 and 547 were away from those areas.

Table 2. Physicochemical characteristics and thermodynamic parameters of VUS with prediction results and clinical data. Folding $\Delta \Delta \mathrm{G}$ : Folding free energy change; ECS: Evolutionary conservation score; RMSF_WT: RMSF of wild-type residue; BC: Breast cancer; GI: Gastro-intestinal cancer.

\begin{tabular}{ccccc}
\hline $\begin{array}{c}\text { MSH2 VUS } \\
\text { Mutations }\end{array}$ & p.Tyr43Cys & p.Ala272Val & p.Asn547Ser & p.Met592Val \\
\hline Folding $\Delta \Delta G$ & -1.228 & -0.788 & -0.26 & -1.286 \\
\hline ECS & 0.767 & 0.877 & 0.863 & 0.575 \\
\hline RMSF_WT & 4.559 & 1.966 & 5.725 & 1.876 \\
\hline Prediction & Non-pathogenic & Pathogenic & Non-pathogenic & Pathogenic \\
\hline BC Mutations & $\begin{array}{c}\text { BRCA2: } \\
\text { c.4936_4939delGAAA }\end{array}$ & N/A & $\begin{array}{c}\text { BRCA2: } \\
\text { c.8791A }>\text { G }\end{array}$ & N/A \\
Clinical feature & $\begin{array}{c}\text { Intermediate } \\
\text { bilateral stage III } \\
\text { size }>1\end{array}$ & $\begin{array}{c}\text { High grade stage I } \\
\text { BC size }<1 \mathrm{~cm}\end{array}$ & $\begin{array}{c}\text { Intermediate, stage } \\
\text { II A size }<1 \mathrm{~cm}\end{array}$ & $\begin{array}{c}\text { Intermediate stage } \\
\text { II A }\end{array}$ \\
\hline $\begin{array}{c}\text { Family History of } \\
\text { BC/GI }\end{array}$ & Yes/Yes & Yes/No & Yes/No & Yes/No \\
\hline
\end{tabular}

The prediction results were compared with Polyphen and SIFT predictions. Polyphen and SIFT predicted p.Ala272Val to be pathogenic, which is consistent with our results, and both of them gave contradictory results on p.Tyr43Cys and p.Met592Val, which were classified as non-pathogenic and pathogenic by our model with the KNN method. However, their predictions were considered not to be reliable since we observed underestimated and overestimated predictions, applying Polyphen/SIFT on the known pathogenic/non-pathogenic mutations (Tables S1-S3).

\subsection{Clinical Features of VUS}

In this work, four VUS were investigated, which were detected in newly diagnosed breast cancer patients from the Clemson University (CU) and Greenwood Genetic Center (GGC) research project. To examine whether those mutations have an impact on breast cancer risk, here we analyzed the outcome of the predictions with corresponding clinical data (Table 2).

Four of 186 patients were identified as MSH2 missense mutation carriers that met the requirements of this study. Genetic test results, mutation type and bio-markers for each carrier were retrieved. To protect the patients' privacy, four carriers were coded as B1, B2, B3 and B4 for this study. The physiochemical features of the VUS were analyzed along with clinical characteristics of the carrier with VUS (Tables S4 and S5).

The B2 patient carried the p.Ala272Val mutation and the B4 patient carried the p.Met592Val mutation. Both mutations were predicted as pathogenic by our model (note that while p.Ala272Val mutation is reported to reduce protein function [40], this mutation is still reported as a mutation with unknown clinical significance based on variant classification guideline by the American College of Medical Genetics and Genomics(ACMG)). Furthermore, previously this mutation was mentioned in a patient with breast cancer, but the patient had p.R3128X in BRCA2 as well [41]). Referring to the clinical information of those two carries, the B2 patient had a high grade, stage I breast cancer, with the size of the tumor smaller than $1 \mathrm{~cm}$. The triple negative bio-markers of estrogen (ER), progesterone (PR) and Her2 complicated the diagnoses. This mutation carrier had a breast cancer family history, but no genetic testing information was included. The p.Met592Val mutation was also predicted to be deleterious. This patient, B4, with the p.Met592Val mutation had intermediate, stage IIA breast cancer with a tumor size larger than $1 \mathrm{~cm}$. The patient was negative for both PR and Her2 markers 
but the estrogen marker was positive. As mentioned, both MSH2 mutation carriers, B2 and B4 were diagnosed with breast cancer, while they did not have mutations in BRCA1,2 genes.

The B1 patient carried the p.Tyr43Cys mutation, which was predicted to be non-pathogenic. This patient carrier was diagnosed with an intermediate, bilateral, stage III breast cancer with positive estrogen and progesterone tumor markers. This B1 mutation carrier also has a family history of BC and GI cancer. When the genetic test result was checked on this carrier, the B1 carrier also had a BRCA2 mutation (NM_000059.3(BRCA2):c.4936_4939delGAAA) which was previously confirmed, by many studies, to be an associated breast cancer risk [42].

The B3 patient carried the p.Asn547Ser mutation, which was predicted to be non-pathogenic. However, this patient had a mutation in the BRCA2 gene (NM_000059.3(BRCA2):c.8791A $>$ G). The carrier was diagnosed with an intermediate, stage IIA breast cancer with a tumor size less than $1 \mathrm{~cm}$. The ER and Her2 tumor markers were both negative for this carrier.

Thus, the pathogenic mutations in MSH2 gene suggested in this study were found in breast cancer patients without variants in $B R C A 1,2$ genes, while the other two patients carrying non-pathogenic mutations in $\mathrm{MSH} 2$ gene had $B R C A 2$ gene variants.

\section{Materials and Methods}

\subsection{Selection of Pathogenic MSH2 Mutations}

The pathogenic mutations investigated in this work were selected from the ClinVar database [24,25]. The search was queried using the search term "MSH2" in the ClinVar database [NM_000251.1] (Figure 3). The results were further refined by using the string "missense mutations". The results consisted of the classifications: Benign (19), likely benign (67), uncertain significance (1238), likely pathogenic (53), pathogenic (40), and conflicting reports of pathogenicity (31) [24,43]. Among them, we selected only mutations that were classified as pathogenic. Furthermore, the mutations with controversial interpretation, or resulting from a single submission were removed from the list, and this resulted in a total of 34 pathogenic mutations.

\subsection{Selection of Benign MSH2 Mutations}

Non-pathogenic (benign) mutations were selected from two sources (Figure 4). One set was obtained from ClinVar Database [24]. The other set was obtained utilizing EXAC [44], the 1000 Genomes Browser [27] and NHLBI-GO Exome Sequencing Project [28]. The explanation of how these two data sets were obtained is provided below.

The search term "MSH2" was used to query the ClinVar database. This resulted in the identification of 19 variants classified as "benign" and 67 variants classified as "likely benign." Excluding those variants with controversial interpretations, the final set of non-pathogenic variants resulted in 20 missense mutations from the ClinVar Database.

The second set of mutations was obtained by using the "MSH2 gene" query within the EXAC browser. The results were further refined by using the "missense mutations" string. In total, 429 missense mutations in the $\mathrm{MSH} 2$ gene were identified. To further identify non-pathogenic mutations delivered from the EXAC database, the 1000 Genomes Project and ESP (NHLBI-GO Exome Sequencing Project) database sets were used to narrow the selections. EXAC database includes the whole genome sequencing data from 60,706 unrelated individuals. Individuals participating in the 1000 Genomes Project were all in healthy conditions, while the ESP database includes cases of genes contributing to heart, lung and blood disorders. Thus, the following two selection criteria were applied on the mutations taken from ClinVar: (a) If a mutation was not detected in the 1000 Genomes Projects, the 1000 Genomes Browser, Ensembl GRCh38 (http://useast.ensembl.org/Homo_sapiens/Info/Index) and (b) if a mutation was present in the ESP database, in both cases the mutation was removed from the list. Thus, 26 mutations were identified as non-pathogenic (benign) mutations. 


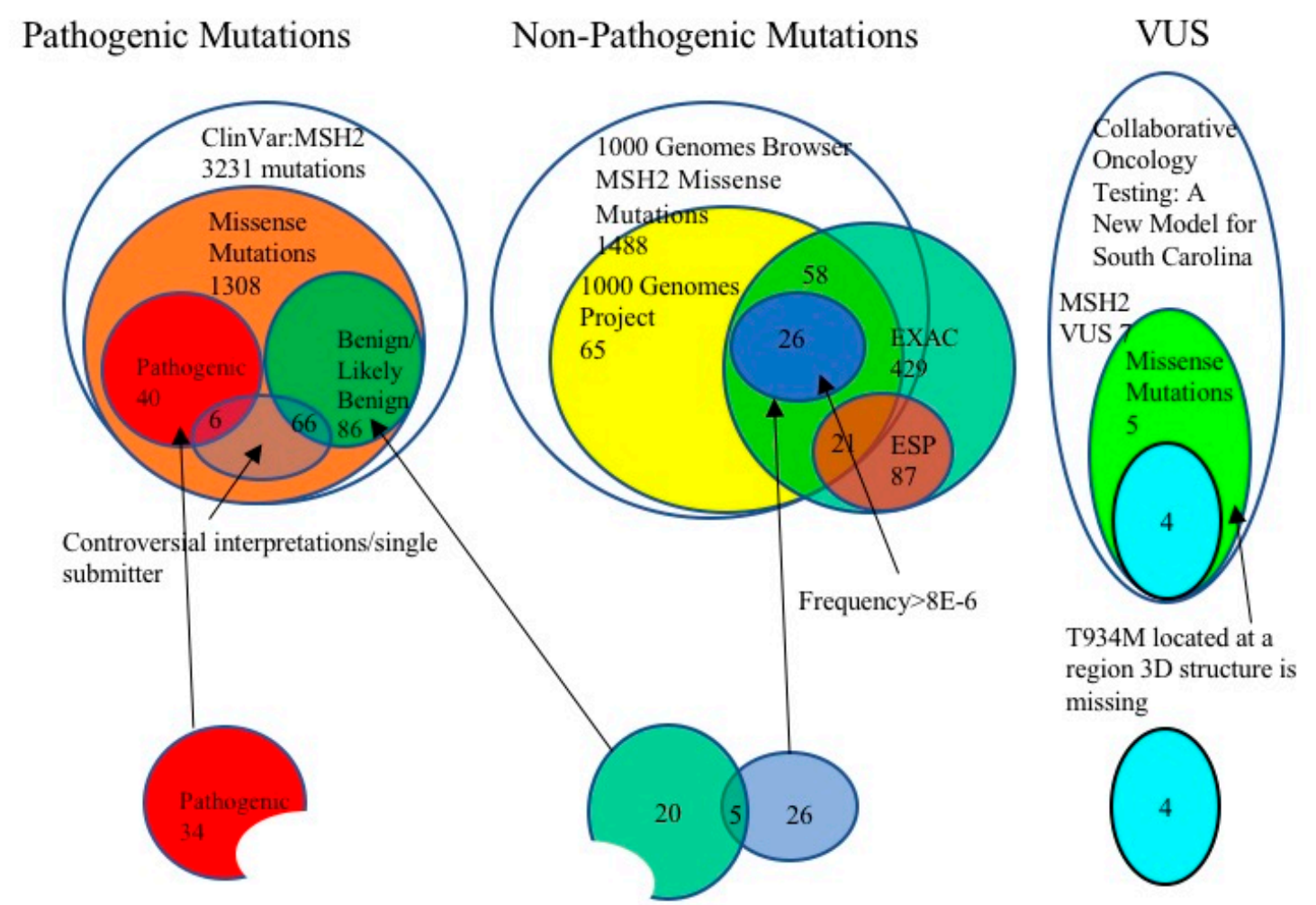

Total Missense mutations:

\section{Pathogenic: 34}

Non-Pathogenic: 41

VUS:4

Figure 4. The selection procedures for pathogenic, non-pathogenic and VUS MSH2 Mutations. Pathogenic mutations were retrieved from the ClinVar database, non-pathogenic mutations were retrieved from the 1000 Genomes project with EXAC database and ESP database; and VUS mutations were from the research project, "Collaborative Oncology Testing: A New Model for South Carolina".

Based on the two sources outlined above, the final set of non-pathogenic mutations was comprised of 41 non-pathogenic missense mutations. Out of them, 20 non-pathogenic mutations were found from the ClinVar and 26 non-pathogenic mutations were found from the 1000 genomes project. Only 5 non-pathogenic mutations were common in ClinVar database and the 1000 genomes project dataset.

\subsection{MSH2 Missense Variants of Uncertain Significance}

Uncertain significance missense variants were identified from the joint research project of the Clemson University School of Nursing and Greenwood Genetic Center entitled "Collaborative Oncology Testing: A New Model for South Carolina" (Figure 4). Institutional review board (IRB) approval for the study was obtained from the Bon Secours Richmond Health System IRB according to 45 CFR 46.111 (see Supplementary Materials: IRB approval documentation).

After being diagnosed with breast cancer between 1 July 2014 and 30 June 2017, a total of 218 patients were recruited from a community cancer center in the upstate of South Carolina. A total of 186 patients provided peripheral blood samples and they were examined by next-generation sequencing [44]. Variants were confirmed with Sanger sequencing and classified according to the American College of Medical Genetics (ACMG) Standards and Guidelines for the Interpretation of Sequence Variants [45]. The ACMG guidelines classifies variants into 5 categories: (1) Benign, (2) likely benign, (3) pathogenic, (4) likely pathogenic, or (5) variant of unknown significance (VUS). 
Seven VUS in the MSH2 gene were found in seven individuals in this group of patients. Since our investigation highly relies on the structure, one of these seven VUS, the VUS p.Thr934Met, is located in a region (residues 855-934) where the 3D structure of MSH2 protein is missing, thus it was excluded from the study. Another two VUSs, c.93C > T and c.2785C $>\mathrm{T}$ were also excluded since one was a synonymous mutation and one was a stop codon (note that the patient with a stop codon had a mutation in BRCA2 gene as well, which is an additional reason not to be included in the analysis). Overall, four VUS missense mutations, p.Tyr43Cys, p.Ala272Val, p.Asn547Ser and p.Met592Val were selected for this study.

\subsection{Preparation of $3 D$ Structure of $M S H 2$}

The crystal structure of the MSH2 protein [NP_000242.1] was obtained from the Protein Data Bank (PDB) [46]. The PDB file (ID: 2O8B) contains chain A, chain B and a short DNA segment, but misses some residues in the structure [21]. Chain A is the MSH2 protein and Chain B is the MSH6 protein. The profix module from the Jackal package was used to rebuild those missing heavy atoms and short loops in the MSH2 and MSH6 protein (Figure 5) [47].

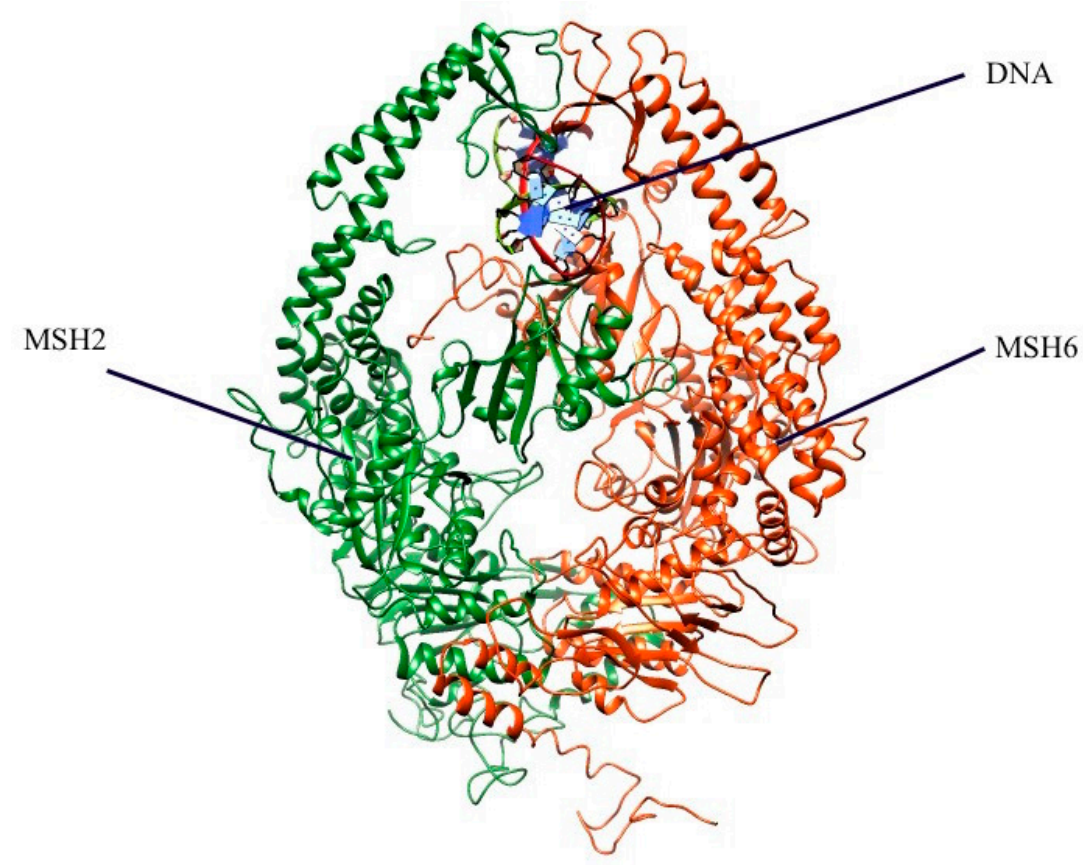

Figure 5. Fixed 3-D structure of the MSH2-MSH6 Complex. The depictions include the proteins for $\mathrm{MSH} 2$ in green and MSH6 in red. The DNA sequence is in blue.

\subsection{Property Distance (PD)}

Peng et al. used property distance (PD) to quantitatively describe the physical-chemical property differences between wild-type and mutant residues [48]. The residue's physical-chemical properties were assessed by a property vector, which included two elements: Hydrophobicity and charge. The experimentally determined hydrophobicity scale was utilized as a reference for the hydrophobicity of residues [49-51]. Regarding the change, residues Arginine (Arg) and Lysine (Lys) carry a +1 charge, aspartic acid (Asp) and glutamic acid (Glu) carry a -1 charge, and all other residues are considered neutral with a charge of 0 . Thus, the property distance between wild-type and mutant residue was calculated using the following equation [48]:

$$
\mathrm{PD}(\mathrm{x}, \mathrm{y})=\sqrt{(\mathrm{H}(\mathrm{x})-\mathrm{H}(\mathrm{y}))^{2}+(\mathrm{Q}(\mathrm{x})-\mathrm{Q}(\mathrm{y}))^{2}}
$$


where $x$ and $y$ represent wild-type and mutant residue, respectively; $H$ and $Q$ represent hydrophobicity and charge for the corresponding residue, respectively.

\subsection{Evolutionary Conservation Score (ECS) Calculations}

The MSH2 sequence of 73 different species were downloaded from UniProt [52]. Multiple sequence alignment was performed with the T-Coffee webserver [52,53]. The ECS of each residue of human MSH2 sequence was calculated with the following equation:

$$
\mathrm{ECS}(\mathrm{i})=\frac{\mathrm{N}(\mathrm{i}) \text { identical }}{\mathrm{N}(\mathrm{i}) \text { total }}
$$

where " $\mathrm{i}$ " is the sequence position of the residue based on human MSH2 sequence. The N(i) identical depicts the number of identical residues for the corresponding location " $\mathrm{i}$ " in the multiple sequence alignment and $\mathrm{N}(\mathrm{i})_{\text {total }}=73$.

\subsection{Folding Free Energy Change $(\Delta \Delta G)$ and Binding Free Energy Change $(\Delta \Delta \Delta G)$}

Several webservers were applied to predict the effect of mutations on protein folding and binding free energies. The webservers used to analyze folding free energy change in this work include DUET [29], I-mutant 3.0 [30], mCSM [31], SAAFEC [32], SDM [33], and PoPMuSiC [34].

The webservers used for determining the binding free energy change in this study included BeAtMuSiC [35], mCSM [31], MutaBind [36], and SAAMBE [37].

\subsection{Relative Solvent Accessible Surface Area (rSASA) and Hydrogen Bond (H-bond) Number Calculations}

The rSASA and H-bonds were calculated using Visual Molecular Dynamics (VMD) [54]. The rSASA for residues were calculated using the following formula:

$$
\operatorname{rSASA}(\mathrm{i})=\frac{\mathrm{SASA}(\mathrm{i})}{\operatorname{SASA}(\mathrm{i}) \max }
$$

where SASA(i) is the solvent accessible surface area calculated for the corresponding residue " $\mathrm{i}$ " in the protein, and SASA(i)max is the maximum possible solvent accessible surface area for the corresponding residue "i" alone.

$\mathrm{H}$-bonds were calculated by the total hydrogen bond number of bonds, being acceptor or donor bonds [55].

$$
\mathrm{N}_{\text {total H-bond(i) }}=\mathrm{N}_{\text {acceptor(i) }}+\mathrm{N}_{\text {donor(i) }}
$$

where $\mathrm{N}_{\text {total H-bond(i) }}$ is the total hydrogen bonds that residue " $\mathrm{i}$ " is involved, which is split into hydrogen bonds that residue " $\mathrm{i}$ " serves as an acceptor $\left(\mathrm{N}_{\text {acceptor(i }}\right)$ and a donor $\left(\mathrm{N}_{\text {donor(i) }}\right)$. The cutoff distance was $3.5 \mathrm{~A}$ and the cutoff angle was 60 degree. The hydrogen bonds were calculated using energy minimized structure.

\subsection{B-Factor of the Alpha Carbonate of the Corresponding Residue}

The X-ray crystallographic B-factor is a parameter which indicates the flexibility of an atom. The B-factor values of the wild-type alpha carbonate $(\mathrm{C} \alpha)$ atoms were extracted from the MSH2 protein crystal structure (PDB ID:2O8B).

\subsection{Molecular Dynamic (MD) Simulations}

Once the missing residues were rebuilt by the profix package, 79 mutant structures (p.Ala2Thr, p.Pro5Gln, p.Pro5Leu, p.Thr8Met, p.Phe23Leu, p.Met26Leu, p.Thr32Ser, p.Tyr43Cys, p.Arg96His, p.Val102Ile, p.Arg106Lys, p.Asn127Ser, p.Met141Val, p.Val161Asp, p.G162Arg, p.Val163Gly, p.Val163Asp, p.Gly164Arg, p.Gly164Glu, p.Asp167His, p.Ile169Val, p.Arg171Lys, p.Leu173Arg, 
p.Leu187Arg, p.Leu187Pro, p.Glu198Gly, p.Cys199Arg, p.Val200Asp, p.Lys228Glu, p.Ser268Leu, p.Ala272Val, p.Val273Ile, p.Leu310Pro, p.Gly322Asp, p.Cys333Tyr, p.G338Glu, p.Pro349Leu, p.Pro349Arg, p.Arg359Ser, p.Leu390Phe, p.Gln419Lys, p.Leu440Pro, p.Arg444Leu, p.Metet453Lys, p.His466Arg, p.Ser479Asn, p.Asn547Ser, p.Ser554Cys, p.Ser554Gly, p.Ser554Thr, p.Thr564Ala, p.Ile577Thr, p.Gly587Arg, p.Met592Val, p.Asp597Ala, p.Pro622Leu, p.Gln629Arg, p.Ala636Pro, p.His639Tyr, p.Ala640Ser, p.Val642Ile, p.Val655Ile, p.Tyr656Cys, p.Glu658Gly, p.Gly669Val, p.G683Arg, p.Met688Ile, p.Met688Arg, p.G692Arg, p.Pro696Leu, p.Cys697Arg, p.Cys697Phe, p.Ile704Thr, p.Gly751Arg, p.Gly759Glu, p.His785Pro, p.Glu809Lys, p.Ala834Thr, p.Lys845Glu) were generated from the wild-type MSH2-MSH6 protein complex structure using the VMD 1.9.3 mutator package [54].

Molecular dynamic (MD) simulation was performed using NAMD2.11 with a Charmm36 force field [56]. The protein structure first underwent energy minimization for 10,000 steps for all simulations in order to relax possible overlaps. Generalized Born implicit solvent (GBIS) was applied in the simulations and the time step was set to $1 \mathrm{fs}$. The temperature in the simulation was set to $300 \mathrm{~K}$. Frame was outputted every 2500 steps in the simulation and the DCD files were subjected to VMD for further analysis. Root mean square deviation (RMSD) of the $\mathrm{C} \alpha$ and root mean square fluctuations (RMSF) of a residue were also calculated using VMD 1.9.3.

\subsection{K-Nearest Neighbors (KNN) and Support Vector Machine (SVM) Classifications}

K-nearest neighbors (KNN) and support vector machine (SVM) algorithms were trained and tested on a total of 75 missense mutations, out of which 34 pathogenic and 41 non-pathogenic. This dataset was randomly split into a training dataset (52 mutations) and a testing dataset ( 23 mutations). The KNN classification was performed using $\mathrm{R}$ studio and optimal $\mathrm{K}$ values were tested to obtain the best performance for the data. The SVM classifier with different kernel was also utilized in R. The training dataset and test dataset were the same as those used in the KNN classification.

\section{Conclusions}

We analyzed the effect of 22 physicochemical characteristics and thermodynamics parameters on wild-type and 75 mutant MSH2 proteins in order to identify the optimal classifiers, which could discriminate pathogenic from non-pathogenic mutations. We found that the wild-type residues corresponding to pathogenic mutations were highly conserved. In contrast, non-pathogenic mutations were located on the positions that had a low evolutionary conservation score. Folding free energy change showed that only non-pathogenic mutations had a value from $-0.5 \mathrm{kcal} / \mathrm{mol}$ to $0.5 \mathrm{kcal} / \mathrm{mol}$. A total of $90 \%$ of wild-type residues where pathogenic mutations were found had a RMSF value between 1-2 $\AA$, while most wild-type residues, where non-pathogenic mutations were found, had a larger RMSF value. These findings indicate that pathogenic $M S H 2$ mutations were in more rigid regions and those mutations affect protein stability, flexibility and conformational dynamics.

In this work, the evolutionary conservation score, folding free energy change and RMSF of wild-type residues were able to discriminate pathogenic from non-pathogenic mutations, thus those three characteristics were applied in the KNN classification model. VUS p.Ala272Val and p.Met592Val were predicted as pathogenic, and VUS p.Tyr43Cys and p.Asn547Ser were predicted as non-pathogenic. The predictions were consistent with clinical observations.

This finding strongly suggests that p.Ala272Val and p.Met592Val in MSH2 genes should be considered for inclusion in genetic testing profiles for breast cancer. Especially for patients without $B R C A 1$ or $B R C A 2$ mutations. However, for such an inclusion, an in vitro validation of mutation pathogenicity is needed and, moreover, the presence of these mutations should be demonstrated in a higher number of patients or in families with BC history. If our findings are confirmed, then this will expand the spectrum of bio-markers and will provide better options for early detection, intervention and diagnosis.

Supplementary Materials: Supplementary materials can be found at http://www.mdpi.com/1422-0067/20/19/ 4828/s1. 
Author Contributions: Conceptualization, E.A., B.W., Y.P. and J.E.; methodology, E.A., P.Y. and B.W.; software, Y.P. and B.W.; validation, B.W., and Y.P.; formal analysis, B.W.; investigation, B.W.; resources, J.E. and E.A.; data curation, B.W.; writing — original draft preparation, B.W.; writing—review and editing, E.A.; visualization, B.W.; supervision, E.A. and J.E.; project administration, E.A.; funding acquisition, E.A.

Funding: This research was supported by NIH, grant numbers R01GM093937 and R01GM125639.

Conflicts of Interest: The authors declare no conflicts of interest.

$\begin{array}{ll}\text { Abbreviations } \\ \text { MMR } & \text { Mismatch Repair } \\ \text { LS } & \text { Lynch Syndrome } \\ \text { EXAC } & \text { Exome Aggregation Consortium } \\ \text { BC } & \text { Breast Cancer } \\ \text { AUC } & \text { Area Under the Curve } \\ \text { RMSD } & \text { Root mean square deviation } \\ \text { RMSF } & \text { Root mean square fluctuations } \\ \text { rSASA } & \text { Relative solvent accessible surface area } \\ \text { MD } & \text { Molecular Dynamics } \\ \text { PD } & \text { Protein Distance } \\ \text { KNN } & \text { K-nearest neighbors } \\ \text { SVM } & \text { Support Vector Machine } \\ \text { WT } & \text { Wild-Type } \\ \text { GI } & \text { Gastro-Intestinal Cancer } \\ \text { VUS } & \text { Variants of Unknown Significance } \\ \text { PMs } & \text { Pathogenic Mutations } \\ \text { NPMs } & \text { Non-Pathogenic Mutations } \\ \text { ESP } & \text { NHLBI-GO Exome Sequencing Project Exome Variant Server } \\ \text { ECS } & \text { Evolutionary Conservation Score }\end{array}$

\section{References}

1. Marti, T.M.; Kunz, C.; Fleck, O. DNA mismatch repair and mutation avoidance pathways. J Cell. Physiol. 2002, 191, 28-41. [CrossRef] [PubMed]

2. Srivatsan, A.; Bowen, N.; Kolodner, R.D. Mispair-specific recruitment of the Mlh1-Pms1 complex identifies repair substrates of the Saccharomyces cerevisiae Msh2-Msh3 complex. J. Biol. Chem. 2014, 289, 9352-9364. [CrossRef] [PubMed]

3. Kolodner, R.D.; Marsischky, G.T. Eukaryotic DNA mismatch repair. Curr. Opin. Genet. Dev. 1999, 9, 89-96. [CrossRef]

4. $\quad$ Lynch, H.T.; Smyrk, T.; Lynch, J.; Fitzgibbons, R., Jr.; Lanspa, S.; McGinn, T. Update on the differential diagnosis, surveillance and management of hereditary non-polyposis colorectal cancer. Eur. J. Cancer 1995, 31, 1039-1046. [CrossRef]

5. Lynch, H.T.; Shaw, M.W.; Magnuson, C.W.; Larsen, A.L.; Krush, A.J. Hereditary factors in cancer. Study of two large midwestern kindreds. Arch. Intern. Med. 1966, 117, 206-212. [CrossRef]

6. Lynch, H.T.; Krush, A.J.; Larsen, A.L. Heredity and endometrial carcinoma. South. Med. J. 1967, 60, $231-235$. [CrossRef] [PubMed]

7. Lynch, H.T.; Krush, A.J. Heredity and adenocarcinoma of the colon. Gastroenterology 1967, 53, 517-527. [CrossRef]

8. Parc, Y.; Boisson, C.; Thomas, G.; Olschwang, S. Cancer risk in 348 French MSH2 or MLH1 gene carriers. J. Med. Genet. 2003, 40, 208-213. [CrossRef]

9. Barrow, E.; Alduaij, W.; Robinson, L.; Shenton, A.; Clancy, T.; Lalloo, F.; Hill, J.; Evans, D.G. Colorectal cancer in HNPCC: Cumulative lifetime incidence, survival and tumour distribution. A report of 121 families with proven mutations. Clin. Genet. 2008, 74, 233-242. [CrossRef]

10. Lynch, H.T.; Coronel, S.M.; Okimoto, R.; Hampel, H.; Sweet, K.; Lynch, J.F.; Barrows, A.; Wijnen, J.; van der Klift, H.; Franken, P.; et al. A founder mutation of the MSH2 gene and hereditary nonpolyposis colorectal cancer in the United States. JAMA 2004, 291, 718-724. [CrossRef] 
11. Janavicius, R.; Elsakov, P. Novel germline MSH2 mutation in lynch syndrome patient surviving multiple cancers. Hered Cancer Clin. Pr. 2012, 10, 1. [CrossRef] [PubMed]

12. Hirano, K.; Yamashita, K.; Yamashita, N.; Nakatsumi, Y.; Esumi, H.; Kawashima, A.; Ohta, T.; Mai, M.; Minamoto, T. Non-Hodgkin's lymphoma in a patient with probable hereditary nonpolyposis colon cancer: Report of a case and review of the literature. Dis. Colon Rectum 2002, 45, 273-279. [CrossRef]

13. Joost, P.; Therkildsen, C.; Dominguez-Valentin, M.; Jonsson, M.; Nilbert, M. Urinary Tract Cancer in Lynch Syndrome; Increased Risk in Carriers of MSH2 Mutations. Urology 2015, 86, 1212-1217. [CrossRef] [PubMed]

14. Provenzale, D.; Gupta, S.; Ahnen, D.J.; Bray, T.; Cannon, J.A.; Cooper, G.; David, D.S.; Early, D.S.; Erwin, D.; Ford, J.M.; et al. Genetic/Familial High-Risk Assessment: Colorectal Version 1.2016, NCCN Clinical Practice Guidelines in Oncology. J. Natl. Compr. Cancer Netw. 2016, 14, 1010-1030. [CrossRef] [PubMed]

15. Whiteside, D.; McLeod, R.; Graham, G.; Steckley, J.L.; Booth, K.; Somerville, M.J.; Andrew, S.E. A homozygous germ-line mutation in the human MSH2 gene predisposes to hematological malignancy and multiple cafe-au-lait spots. Cancer Res. 2002, 62, 359-362. [PubMed]

16. Zhu, M.; Chen, H.M.; Wang, Y.P. Missense mutations of MLH1 and MSH2 genes detected in patients with gastrointestinal cancer are associated with exonic splicing enhancers and silencers. Oncol. Lett. 2013, 5, 1710-1718. [CrossRef]

17. Takano, K.; Ichikawa, Y.; Ueno, E.; Ohwada, M.; Suzuki, M.; Tsunoda, H.; Miwa, M.; Uchida, K.; Yoshikawa, H. Microsatellite instability and expression of mismatch repair genes in sporadic endometrial cancer coexisting with colorectal or breast cancer. Oncol. Rep. 2005, 13, 11-16. [CrossRef]

18. Lacroix-Triki, M.; Lambros, M.B.; Geyer, F.C.; Suarez, P.H.; Reis-Filho, J.S.; Weigelt, B. Absence of microsatellite instability in mucinous carcinomas of the breast. Int. J. Clin. Exp. Pathol. 2010, 4, 22-31.

19. Goldberg, M.; Bell, K.; Aronson, M.; Semotiuk, K.; Pond, G.; Gallinger, S.; Zbuk, K. Association between the Lynch syndrome gene MSH2 and breast cancer susceptibility in a Canadian familial cancer registry. J. Med. Genet. 2017, 54, 742-746. [CrossRef]

20. Rashid, M.U.; Naeemi, H.; Muhammad, N.; Loya, A.; Yusuf, M.A.; Lubinski, J.; Jakubowska, A.; Hamann, U. A novel deleterious c.2656G > T MSH2 germline mutation in a Pakistani family with a phenotypic overlap of hereditary breast and ovarian cancer and Lynch syndrome. Hered Cancer Clin. Pract. 2016, 14, 14. [CrossRef]

21. Warren, J.J.; Pohlhaus, T.J.; Changela, A.; Iyer, R.R.; Modrich, P.L.; Beese, L.S. Structure of the human MutSalpha DNA lesion recognition complex. Mol. Cell 2007, 26, 579-592. [CrossRef] [PubMed]

22. Mazur, D.J.; Mendillo, M.L.; Kolodner, R.D. Inhibition of Msh6 ATPase activity by mispaired DNA induces a Msh2(ATP)-Msh6(ATP) state capable of hydrolysis-independent movement along DNA. Mol. Cell 2006, 22, 39-49. [CrossRef] [PubMed]

23. Ollila, S.; Sarantaus, L.; Kariola, R.; Chan, P.; Hampel, H.; Holinski-Feder, E.; Macrae, F.; Kohonen-Corish, M.; Gerdes, A.M.; Peltomaki, P.; et al. Pathogenicity of MSH2 missense mutations is typically associated with impaired repair capability of the mutated protein. Gastroenterology 2006, 131, 1408-1417. [CrossRef] [PubMed]

24. Landrum, M.J.; Lee, J.M.; Benson, M.; Brown, G.; Chao, C.; Chitipiralla, S.; Gu, B.; Hart, J.; Hoffman, D.; Hoover, J.; et al. ClinVar: Public archive of interpretations of clinically relevant variants. Nucleic Acids Res. 2016, 44, D862-D868. [CrossRef] [PubMed]

25. Landrum, M.J.; Lee, J.M.; Riley, G.R.; Jang, W.; Rubinstein, W.S.; Church, D.M.; Maglott, D.R. ClinVar: Public archive of relationships among sequence variation and human phenotype. Nucleic Acids Res. 2014, 42, D980-D985. [CrossRef]

26. Taverna, P.; Liu, L.; Hanson, A.J.; Monks, A.; Gerson, S.L. Characterization of MLH1 and MSH2 DNA mismatch repair proteins in cell lines of the NCI anticancer drug screen. Cancer Chemother. Pharmacol. 2000, 46, 507-516. [CrossRef]

27. Genomes Project, C.; Auton, A.; Brooks, L.D.; Durbin, R.M.; Garrison, E.P.; Kang, H.M.; Korbel, J.O.; Marchini, J.L.; McCarthy, S.; McVean, G.A.; et al. A global reference for human genetic variation. Nature 2015, 526, 68-74.

28. ESP. NHLBI-GO Exome Sequencing Project Exome Variant Server; ESP (Exome Sequencing Project): Seattle, WA, USA, 2017.

29. Pires, D.E.; Ascher, D.B.; Blundell, T.L. DUET: A server for predicting effects of mutations on protein stability using an integrated computational approach. Nucleic Acids Res. 2014, 42, W314-W319. [CrossRef]

30. Capriotti, E.; Altman, R.B. Improving the prediction of disease-related variants using protein three-dimensional structure. BMC Bioinform. 2011, 12, S3. [CrossRef] 
31. Pires, D.E.; Ascher, D.B.; Blundell, T.L. mCSM: Predicting the effects of mutations in proteins using graph-based signatures. Bioinformatics 2014, 30, 335-342. [CrossRef]

32. Getov, I.; Petukh, M.; Alexov, E. SAAFEC: Predicting the Effect of Single Point Mutations on Protein Folding Free Energy Using a Knowledge-Modified MM/PBSA Approach. Int. J. Mol. Sci. 2016, 17, 512. [CrossRef] [PubMed]

33. Topham, C.M.; Srinivasan, N.; Blundell, T.L. Prediction of the stability of protein mutants based on structural environment-dependent amino acid substitution and propensity tables. Prot. Eng. 1997, 10, 7-21. [CrossRef] [PubMed]

34. Dehouck, Y.; Kwasigroch, J.M.; Gilis, D.; Rooman, M. PoPMuSiC 2.1: A web server for the estimation of protein stability changes upon mutation and sequence optimality. BMC Bioinform. 2011, 12, 151. [CrossRef] [PubMed]

35. Dehouck, Y.; Kwasigroch, J.M.; Rooman, M.; Gilis, D. BeAtMuSiC: Prediction of changes in protein-protein binding affinity on mutations. Nucleic Acids Res. 2013, 41, W333-W339. [CrossRef] [PubMed]

36. Li, M.; Simonetti, F.L.; Goncearenco, A.; Panchenko, A.R. MutaBind estimates and interprets the effects of sequence variants on protein-protein interactions. Nucleic Acids Res. 2016, 44, W494-W501. [CrossRef] [PubMed]

37. Petukh, M.; Dai, L.; Alexov, E. SAAMBE: Webserver to Predict the Charge of Binding Free Energy Caused by Amino Acids Mutations. Int. J. Mol. Sci. 2016, 17, 547. [CrossRef] [PubMed]

38. Kucukkal, T.G.; Petukh, M.; Li, L.; Alexov, E. Structural and physico-chemical effects of disease and non-disease nsSNPs on proteins. Curr. Opin. Struct. Biol. 2015, 32, 18-24. [CrossRef]

39. Petukh, M.; Kucukkal, T.G.; Alexov, E. On human disease-causing amino acid variants: Statistical study of sequence and structural patterns. Hum. Mutat. 2015, 36, 524-534. [CrossRef]

40. Nilbert, M.; Wikman, F.P.; Hansen, T.V.; Krarup, H.B.; Orntoft, T.F.; Nielsen, F.C.; Sunde, L.; Gerdes, A.M.; Cruger, D.; Timshel, S.; et al. Major contribution from recurrent alterations and MSH6 mutations in the Danish Lynch syndrome population. Fam. Cancer 2009, 8, 75-83. [CrossRef]

41. Dominguez-Valentin, M.; Evans, D.G.R.; Nakken, S.; Tubeuf, H.; Vodak, D.; Ekstrom, P.O.; Nissen, A.M.; Morak, M.; Holinski-Feder, E.; Martins, A.; et al. Genetic variants of prospectively demonstrated phenocopies in BRCA1/2 kindreds. Hered Cancer Clin. Pract. 2018, 16, 4. [CrossRef]

42. Borg, A.; Haile, R.W.; Malone, K.E.; Capanu, M.; Diep, A.; Torngren, T.; Teraoka, S.; Begg, C.B.; Thomas, D.C.; Concannon, P.; et al. Characterization of BRCA1 and BRCA2 deleterious mutations and variants of unknown clinical significance in unilateral and bilateral breast cancer: The WECARE study. Hum. Mutat 2010, 31, E1200-E1240. [CrossRef] [PubMed]

43. ClinVar. Available online: https://www.ncbi.nlm.nih.gov/clinvar/ (accessed on 14 November 2017).

44. Lek, M.; Karczewski, K.J.; Minikel, E.V.; Samocha, K.E.; Banks, E.; Fennell, T.; O’Donnell-Luria, A.H.; Ware, J.S.; Hill, A.J.; Cummings, B.B.; et al. Analysis of protein-coding genetic variation in 60,706 humans. Nature 2016, 536, 285-291. [CrossRef] [PubMed]

45. Richards, S.; Aziz, N.; Bale, S.; Bick, D.; Das, S.; Gastier-Foster, J.; Grody, W.W.; Hegde, M.; Lyon, E.; Spector, E.; et al. Standards and guidelines for the interpretation of sequence variants: A joint consensus recommendation of the American College of Medical Genetics and Genomics and the Association for Molecular Pathology. Genet. Med. 2015, 17, 405-424. [CrossRef] [PubMed]

46. Rose, P.W.; Beran, B.; Bi, C.; Bluhm, W.F.; Dimitropoulos, D.; Goodsell, D.S.; Prlic, A.; Quesada, M.; Quinn, G.B.; Westbrook, J.D.; et al. The RCSB Protein Data Bank: Redesigned web site and web services. Nucleic Acids Res. 2011, 39, D392-D401. [CrossRef] [PubMed]

47. Petrey, D.; Xiang, Z.; Tang, C.L.; Xie, L.; Gimpelev, M.; Mitros, T.; Soto, C.S.; Goldsmith-Fischman, S.; Kernytsky, A.; Schlessinger, A.; et al. Using multiple structure alignments, fast model building, and energetic analysis in fold recognition and homology modeling. Proteins 2003, 53, 430-435. [CrossRef] [PubMed]

48. Peng, Y.; Myers, R.; Zhang, W.; Alexov, E. Computational Investigation of the Missense Mutations in DHCR7 Gene Associated with Smith-Lemli-Opitz Syndrome. Int. J. Mol. Sci 2018, 19, 141. [CrossRef] [PubMed]

49. Wimley, W.C.; White, S.H. Experimentally determined hydrophobicity scale for proteins at membrane interfaces. Nat. Struct. Biol. 1996, 3, 842-848. [CrossRef]

50. Petukh, M.; Li, M.; Alexov, E. Predicting Binding Free Energy Change Caused by Point Mutations with Knowledge-Modified MM/PBSA Method. PLoS Comput. Biol. 2015, 11, e1004276. [CrossRef] [PubMed] 
51. Peng, Y.; Sun, L.; Jia, Z.; Li, L.; Alexov, E. Predicting protein-DNA binding free energy change upon missense mutations using modified MM/PBSA approach: SAMPDI webserver. Bioinformatics 2018, 34, 779-786. [CrossRef]

52. UniProt, C. UniProt: A hub for protein information. Nucleic Acids Res. 2015, 43, D204-D212.

53. Notredame, C.; Higgins, D.G.; Heringa, J. T-Coffee: A novel method for fast and accurate multiple sequence alignment. J. Mol. Biol. 2000, 302, 205-217. [CrossRef] [PubMed]

54. Humphrey, W.; Dalke, A.; Schulten, K. VMD: Visual molecular dynamics. J. Mol. Gr. 1996, 14, 33-38. [CrossRef]

55. Wu, B.; Eggert, J.; Alexov, E. Molecular mechanisms underlying pathogenic missense mutations. eLS 2014. [CrossRef]

56. Phillips, J.C.; Braun, R.; Wang, W.; Gumbart, J.; Tajkhorshid, E.; Villa, E.; Chipot, C.; Skeel, R.D.; Kale, L.; Schulten, K. Scalable molecular dynamics with NAMD. J. Comput. Chem. 2005, 26, 1781-1802. [CrossRef] [PubMed]

(C) 2019 by the authors. Licensee MDPI, Basel, Switzerland. This article is an open access article distributed under the terms and conditions of the Creative Commons Attribution (CC BY) license (http://creativecommons.org/licenses/by/4.0/). 